\title{
Effects of Kalsis, A Dietary Supplement, on Bone Metabolism in the Ovariectomized Rats
}

\author{
Mercedes Montero, ${ }^{1}$ Manuel Díaz-Curiel, ${ }^{2}$ David Guede, ${ }^{3}$ Jose Ramón Caeiro, ${ }^{4}$ \\ Marta Martín-Fernández, ${ }^{1}$ Mercedes Rubert, ${ }^{1}$ Daisy Navarro, ${ }^{5}$ and Concepción de la Piedra ${ }^{1}$ \\ ${ }^{1}$ Bioquímica Investigación, Instituto de Investigación Sanitaria, Fundación Jiménez Díaz, Avenida Reyes Católicos 2, \\ 28040 Madrid, Spain \\ ${ }^{2}$ Internal Medicine, Instituto de Investigación, Sanitaria Fundación Jiménez Díaz, 28040 Madrid, Spain \\ ${ }^{3}$ Trabeculae Technology Based Firm, Technological Park of Galicia, 32900 Ourense, Spain \\ ${ }^{4}$ Traumatology and Orthopedic Surgery, University Hospital Complex of Santiago de Compostela, \\ 15706 Santiago de Compostela, Spain \\ ${ }^{5}$ Instituto Nacional de Endocrinología, 10400 La Habana, Cuba \\ Correspondence should be addressed to Concepción de la Piedra, cpiedra@fjd.es
}

Received 4 June 2012; Revised 1 August 2012; Accepted 11 September 2012

Academic Editor: Hope Weiler

Copyright (C 2012 Mercedes Montero et al. This is an open access article distributed under the Creative Commons Attribution License, which permits unrestricted use, distribution, and reproduction in any medium, provided the original work is properly cited.

\begin{abstract}
We studied the ability of Kalsis, a food supplement that contains selenium, citric acid, and vitamin E, to prevent the effects of ovariectomy on bone loss. Six-month-old, Wistar female rats were studied. Groups $(n=12)$ : SHAM: sham-operated rats; OVX: ovariectomized rats, treated with vehicle; OVX + Kalsis: ovariectomized rats treated with Kalsis ( $25 \mathrm{mg} / \mathrm{kg} /$ day) for 3 months. Bone mineral density (BMD) was determined by DXA in lumbar spine and femur. Computerized microtomography $(\mu \mathrm{CT})$ in femur and serum osteocalcin (BGP), aminoterminal propeptide of procollagen I (PINP), $\beta$-isomer of carboxyterminal telopeptide of collagen I (CTX), and 5b isoenzyme of tartrate-resistant acid phosphatase (TRAP) were performed. Treatment with Kalsis prevented BMD loss in OVX group. $\mu \mathrm{CT}$ showed a decrease in BV/TV, and trabecular number, and an increase in trabecular separation in OVX rats. Kalsis administration attenuated partially bone loss observed by $\mu \mathrm{CT}$ due to ovariectomy. BGP, PINP, and the resorption index (CTX/TRAP) were increased in OVX group. Treatment with Kalsis maintained this increase. The mechanism of action of this supplement is not through a decrease in bone remodelling rate. The antioxidant action of this food supplement, due to the synergism of all its components, as a cause of its beneficial effect is suggested.
\end{abstract}

\section{Introduction}

Osteoporosis was defined as a skeletal disease characterized by low bone mineral density (BMD) and poor bone quality that reduces bone strength and increases the risk of fractures [1]. Osteoporosis is a disease with different etiologies: several pharmacological treatments, aging, and so forth, but the most important group, due to the number of affected patients, is postmenopausal osteoporosis. The fact that estrogen lack affects bone remodelling leads to an increase of bone resorption over formation, with the corresponding bone loss [2-5].

Recent animal studies have identified additional unexpected regulatory effects of estrogen centered at the level of adaptative immune response [6]. Furthermore, a potential role of estrogens for reactive oxygen species (ROS) has now been identified in both human and animals [6]. It is known that oxidative stress is an important mediator of bone loss in postmenopausal osteoporosis by generating a more oxidized bone microenvironment $[7,8]$. In vivo support of this hypothesis is found from experiments in which OVX induces oxidative stress and impairs antioxidant expression in adult rats [9]. In an interesting review about estrogen deficiency and bone loss, Weitzmann and Pacifici [6] suggest a model for OVX-induced bone loss in which estrogen deficiency lowers antioxidant levels, thereby increasing ROS. A decrease in plasma antioxidants was found in aged osteoporotic women [8]. Osteoporosis increased oxidative stress in severe 
osteoporotic syndrome in young males (mean of 33 years of age) $[10]$.

The antioxidants can be endogenous or obtained exogenously, for example, as a part of diet or as dietary supplements. The most efficient enzymatic antioxidants involve glutathione peroxidase and catalase. Nonenzymatic antioxidants include vitamins $\mathrm{E}$ and $\mathrm{C}$ (ascorbic acid), carotenoids, and other compounds [11]. Glutathione peroxidase, responsible for intracellular degradation of hydrogen peroxide, is the predominant antioxidant enzyme expressed by osteoclasts [12] and is upregulated by estrogen. Although it cannot be classified as an antioxidant, selenium is an important cofactor that binds to the catalytic site of an apoenzyme, rendering it active [11]. Its protective effects appear to be associated with its presence in the multiform of glutathione peroxidases, which are known to protect DNA and other cellular damage from oxidative stress $[13,14]$. The retarded growth induced by selenium deficiency in rats is associated with osteopenia [15].

Drugs used to prevent and treat postmenopausal osteoporosis have been designed to act directly on bone remodelling comprising their main intended effect to maintain or recover bone mass [16]. They can be classified into three main groups: resorption inhibitors such as calcitonin, raloxifene, and bisphosphonates; bone formation stimulators like parathyroid hormone; those which produce both effects simultaneously, such as strontium ranelate. All these pharmacological treatments have been shown to be effective either in increasing bone mineral density (BMD) and/or reducing fracture rates [17-19]. However, their long-term use is currently a controversial subject within the scientific community. Some researchers have directed their efforts to the aspect of antioxidant activity. As a result of such efforts, a positive correlation has been established between intake of antioxidants and bone mass [20]. Under this concept the potential protective mechanisms of carotenoids [21] or green tea polyphenols $[22,23]$ as antioxidant agents preventing bone loss have been investigated. Ascorbic acid intake (antioxidant) increases BMD in postmenopausal women [24].

Kalsis (Catalysis Lab., Spain) is an antioxidant, a dietary supplement that contains, among others, vitamins $\mathrm{C}$ and $\mathrm{E}$ and an organic selenium compound. Previous studies in humans seem to demonstrate its beneficial effects on bone mass in osteoporotic patients (unpublished results). Due to the inherent difficulties associated with human investigation, the use of animal models is a helpful tool. The ovariectomized rat is a widely validated experimental model for studying postmenopausal osteoporosis and the effects produced by the different drugs used to prevent or treat the disease [25]. The aim of this study was to examine the effectiveness of Kalsis in preventing bone loss caused by removal of ovaries in rats when administered immediately after ovariectomy.

\section{Materials and Methods}

2.1. Animals. Thirty-six female Wistar rats from the stabulary of Instituto de Investigación Sanitaria Fundación
Jiménez Díaz (Madrid, Spain) with 6 months of age and weighing $261.7 \pm 19.0 \mathrm{~g}$ were ovariectomized or sham operated using Ketamine (40 mg/kg, Ketolar, Bayer) and Xilacine $(8 \mathrm{mg} / \mathrm{kg}$, Rompún, Parke-Davis, Pfizer). After that, the rats were randomized in the following groups $(n=$ 12 per group): SHAM group treated with vehicle (water); ovariectomized group also treated with vehicle (OVX); ovariectomized group treated with Kalsis $(25 \mathrm{mg} / \mathrm{kg} /$ day $)$ (OVX + K25) for three months. This dose, by $\mathrm{kg}$ of body weight, is the same as that recommended for humans in the commercial insert of this compound. The animals were kept under constant conditions $\left(22^{\circ} \mathrm{C}, 12\right.$ hours per day lightdark cycles), and food (standard laboratory chow) and water were offered ad libitum. The diet included Ca $(8400 \mathrm{mg} / \mathrm{kg})$, P (5700 mg/kg), and Vit D (900 UI/kg).

On the day following the last treatment, the experimental animals were weighed and killed by exsanguination under ether anaesthesia. Blood samples were obtained by cardiac puncture and serum samples were immediately frozen as aliquots at $-80^{\circ} \mathrm{C}$. The animals were frozen at $-20^{\circ} \mathrm{C}$ and maintained thawed at $4^{\circ} \mathrm{C}$ for two days before the analysis.

All procedures were carried out in accordance with European Community Standards on the Care and Use of Laboratory Animals.

2.2. Treatment. Kalsis was kindly supplied by Catalysis Laboratories, Spain. The exact composition of the supplement is (per $500 \mathrm{mg}$ capsule): calcium gluconate (185 mg), magnesium gluconate $(85 \mathrm{mg})$, citric fibre $(70 \mathrm{mg})$, Lactobacillus acidophilus $(51 \mathrm{mg})$, vitamin C (20 mg), vitamin E ( $3 \mathrm{mg})$, and selenium-rich yeast $(16 \mathrm{mg}$ ) (between 1 and $1.2 \mu \mathrm{g}$ of selenium/mg of yeast). Each component of the formula was activated as indicated by the manufacturer [26]. Kalsis was resuspended in $0.3 \mathrm{~mL}$ of distilled water and administered daily by oral gavage; $0.3 \mathrm{~mL}$ of water/rat/day was administered as a vehicle. Treatment in this study began the day after surgery.

2.3. Biochemical Parameters. Serum calcium levels were measured using an autoanalyzer method (modular Roche DDPP). The sensitivity of this method was $0.2 \mathrm{mg} / \mathrm{dL}$. The intra- and interassay coefficients of variation were $<0.9 \%$ and $<1.5 \%$, respectively.

Serum creatinine levels were measured using an autoanalyzer method (modular Roche DDPP). The sensitivity of this method was $0.1 \mathrm{mg} / \mathrm{dL}$. Intra- and interassay coefficients of variation were $<0.7 \%$ and $<2.3 \%$, respectively.

Serum bone Glaprotein (BGP) was measured by a specific ELISA for rat BGP (Rat-Mid Osteocalcin, IDS, UK). The sensitivity of this assay was $50.0 \mathrm{ng} / \mathrm{mL}$. Intra- and interassay coefficients of variation were $<5.0 \%$ and $<5.5 \%$, respectively.

Serum N-terminal propeptide of type I procollagen (PINP) was measured by a specific ELISA for both rat and mouse PINP (Rat/Mouse PINP EIA, IDS, UK). The sensitivity of this assay was $0.7 \mathrm{ng} / \mathrm{mL}$. The intra- and interassay coefficients of variation were $<7.4 \%$ and $<8.0 \%$, respectively. 
Beta isomer of serum C-telopeptide of type I collagen (CTX) was measured by an ELISA specific for rat CTX (RatLaps ELISA, IDS, UK). The sensitivity of the assay was $2.0 \mathrm{ng} / \mathrm{mL}$. Intra- and interassay variation coefficients were $<5.6 \%$ and $<10.5 \%$, respectively.

Serum tartrate-resistant acid phosphatase form $5 b$ (TRAP 5b) was measured by an ELISA specific for rat TRAP 5b (RatTRAP Assay, IDS, UK). The sensitivity of the assay was $0.1 \mathrm{U} / \mathrm{L}$. The intra- and interassay variation coefficients were $<4.1 \%$ and $<3.0 \%$, respectively.

2.4. Bone Mineral Density. Bone mineral density (BMD) was determined in situ in the lumbar spine (L2, L3, and L4) and in the whole left femur by DEXA (dual energy Xray densitometry) using a HOLOGIC QDR-1000 TM (S/N 277) (Hologic, Inc., Waltham, MA, USA) with small-animal software [27]. The whole left femur was previously extracted and cleaned of adjacent tissue. The intraassay and interassay variation coefficients were $<5.3 \%$ and $<1.2 \%$, respectively. The scans of the femur were studied in order to assess the BMD of the whole femur and the scans of the L2, L3, and L4 vertebrae were analyzed for BMD of the whole vertebrae.

2.5. 3D Trabecular Microarchitecture Analysis of Femur by Micro-CT. The distal region of the right femur was thawed at room temperature and immersed in saline solution $(0.9 \%$ $\mathrm{NaCl}$ ) and analyzed, without further sample preparation, by micro-CT (Skyscan N.V., Aartselaar, Belgium), using an Xray tube voltage of $100 \mathrm{kV}$ and current of $100 \mu \mathrm{A}$ and with a $1.0 \mathrm{~mm}$ aluminium filter. The scanning angular rotation was $185^{\circ}$, and the angular increment was $0.45^{\circ}$. The voxel size was $11.0 \mu \mathrm{m}$. Data sets were reconstructed using a modified Feldkamp algorithm [28] and segmented into binary images (8-bit BMP images) using adaptive local thresholding. For analysis of the microarchitectural properties of trabecular and cortical bone regions, femora specimens were evaluated within a conforming volume of interest (VOI). In the case of the trabecular femur region, a VOI was selected starting at a distance of $1.00 \mathrm{~mm}$ from the growth plate (GP), and extending a longitudinal distance of $2.50 \mathrm{~mm}$ in the proximal direction (226 image slices analyzed, cortical bone excluded).

Both trabecular and cortical bone regions were obtained by tracing regions of interest and then analyzed using the commercial software provided with the equipment (SkyScan CT-analyzer software, version 1.7.0). Morphometric indices of the trabecular bone region were determined from the microtomographic data sets (within a VOI) using direct 3D morphometry. Total volume of VOI (tissue volume TV, $\left.\mathrm{mm}^{3}\right)$ and trabecular bone volume $\left(\mathrm{BV} ; \mathrm{mm}^{3}\right)$ were calculated based on the hexahedral marching cubes volume model of the VOI. Trabecular bone volume (BV/TV; \%) was directly calculated. Trabecular thickness (Tb.Th; $\mathrm{mm}$ ), trabecular separation (Tb.Sp; $\mathrm{mm}$ ), and trabecular number (Tb.N; $1 / \mathrm{mm}$ ) were measured directly with the use of $3 \mathrm{D}$ images using methods previously described [29, 30]. Measurements of trabecular thickness were calibrated by scanning and analyzing three aluminum foils with thicknesses of 50, 125 and $250 \mu \mathrm{m}$. The trabecular bone pattern factor (Tb.Pf;
TABLe 1: Creatinine, calcium, CTX/TRAP index, BGP, and PINP serum levels in female Wistar rats at the end of the study. Sixmonth-old rats at the beginning of the study were ovariectomized (OVX) or sham-operated (SHAM) and randomized in the following groups: SHAM group (SHAM) treated with vehicle for 3 months; ovariectomized group (OVX) treated also with vehicle for 3 months; ovariectomized group treated with Kalsis at a dose of $25 \mathrm{mg} / \mathrm{kg} /$ day, for 3 months (OVX + K25). Statistical significance $P<0.05$ : a versus SHAM.

\begin{tabular}{lccc}
\hline & SHAM & OVX & OVX + K25 \\
\hline Creatinine $(\mathrm{mg} / \mathrm{dL})$ & $0.66 \pm 0.04$ & $0.71 \pm 0.13$ & $0.65 \pm 0.03$ \\
Calcium $(\mathrm{mg} / \mathrm{dL})$ & $9.2 \pm 0.05$ & $9.2 \pm 0.4$ & $9.1 \pm 0.2$ \\
CTX/TRAP index & $2.6 \pm 1.1$ & $12.5 \pm 4.5^{\mathrm{a}}$ & $16.5 \pm 5.6^{\mathrm{a}}$ \\
BGP $(\mathrm{ng} / \mathrm{mL})$ & $67.4 \pm 1.1$ & $128.2 \pm 23.8^{\mathrm{a}}$ & $149.9 \pm 33.4^{\mathrm{a}}$ \\
PINP $(\mathrm{ng} / \mathrm{mL})$ & $4.8 \pm 1.1$ & $7.5 \pm 2.8^{\mathrm{a}}$ & $7.2 \pm 1.4^{\mathrm{a}}$ \\
\hline
\end{tabular}

$1 / \mathrm{mm}$ ) was also calculated using the direct $3 \mathrm{D}$ model. The trabecular bone pattern factor (Tb.Pf; $1 / \mathrm{mm}$ ) measures the relative convexity or concavity of the total bone surface [31]. The coefficient of variation $(\% \mathrm{CV})$ values for all these measurements were $<5 \%$.

2.6. Statistical Analyses. The results of the experiments were expressed as the mean \pm SD of the different parameters. A nonparametric method, the Mann-Whitney test (Medcalc Software Program, Belgium), was used to compare the different treatment groups. A $P$ value $<0.05$ was accepted as denoting a significant difference.

\section{Results}

3.1. Body Weight Gain. Three months after surgery, rats in the OVX group showed higher body weight when compared with SHAM group $(348 \pm 25$ versus $285 \pm 30 \mathrm{~g}, P<0.001)$, according to previous reports [32]. OVX + K25 group presented also higher body weight than SHAM group (320 \pm 36 versus $285 \pm 30, P<0.05)$. Body weight gain was higher in the OVX rats compared with the OVX + K25 $(P<0.05)$. Kalsis partially prevented weight gain, which was lower than that of the OVX group, although higher than that of the SHAM rats.

3.2. Biochemical Parameters. Table 1 shows values of serum creatinine, calcium, and the biochemical markers of bone turnover CTX/TRAP (resorption), BGP, and PINP (formation) in all the groups studied. The levels of creatinine and calcium fell within the normal range in the three groups of rats. As expected, ovariectomy produced a significant increase in bone remodelling 3 months after surgery, both in terms of resorption (CTX/TRAP index) and formation (BGP and PINP). Kalsis administration immediately after ovariectomy (OVX + K25) maintained the increase in bone remodelling produced by ovaries removing; the levels of CTX/TRAP index, BGP, and PINP in rats treated with Kalsis were similar to those of ovariectomized animals without any treatment (Table 1). 
TABle 2: Bone Area (BA), bone mineral content (BMC), and bone mineral density (BMD) measured in femur and in lumbar vertebra in female Wistar rats at the end of the study. Six-month-old rats at the beginning of the study, ovariectomized (OVX) or shamoperated (SHAM) were randomized in the following groups: SHAM group (SHAM), treated with vehicle for 3 months; ovariectomized group (OVX) also treated with vehicle for 3 months; ovariectomized group treated with Kalsis at a dose of $25 \mathrm{mg} / \mathrm{kg} / \mathrm{day}$, for 3 months (OVX + K25). Statistical significance $P<0.05$ : a versus SHAM; b versus OVX.

\begin{tabular}{lccc}
\hline & BA $\left(\mathrm{cm}^{2}\right)$ & BMC $(\mathrm{g})$ & BMD $\left(\mathrm{cm}^{2} / \mathrm{g}\right)$ \\
\hline SHAM & $1.730 \pm 0.15$ & $0.485 \pm 0.072$ & $0.280 \pm 0.017$ \\
OVX & $1.869 \pm 0.069^{\mathrm{a}}$ & $0.474 \pm 0.074$ & $0.263 \pm 0.011^{\mathrm{a}}$ \\
OVX + K25 & $1.847 \pm 0.122^{\mathrm{a}}$ & $0.506 \pm 0.05$ & $0.273 \pm 0.011^{\mathrm{b}}$ \\
\hline \multicolumn{3}{c}{ Lumbar } \\
\hline SHAM & $1.423 \pm 0.127$ & $0.326 \pm 0.054$ & $0.235 \pm 0.021$ \\
OVX & $1.499 \pm 0.104$ & $0.324 \pm 0.038$ & $0.216 \pm 0.013^{\mathrm{a}}$ \\
OVX + K25 & $1.526 \pm 0.123^{\mathrm{a}}$ & $0.347 \pm 0.053$ & $0.227 \pm 0.017^{\mathrm{b}}$ \\
\hline
\end{tabular}

3.3. Bone Mineral Density. As expected, 3 months after ovariectomy the OVX rats presented a significant decrease in femoral BMD and lumbar BMD. Daily treatment with Kalsis for 3 months avoided the loss in femoral and lumbar BMD due to ovariectomy, and the levels of BMD in the treated group were similar to those of the SHAM group. The decrease in femoral BMD in OVX group is due mainly to an increase in BA. Although there is also an increase in BA in the OVX + K25 group, the increase in BMC with respect to OVX group leads to a higher value of BMD than that of OVX group. In the case of lumbar BMD a similar feature is observed (Table 2).

3.4. Computed Microtomography. Figure 1 shows representative images of bone trabecular 3D-microarchitecture in femur sections of all the study groups as obtained by computed microtomography.

As shown in Table 3, which provides the results of 3D trabecular microarchitecture analysis of femur by microtomography, the OVX rats underwent a significant decrease in BV/TV values and in number of trabeculae (Tb.N) with respect to the SHAM group. Likewise, separation of trabeculae (Tb.Sp) was significantly higher with respect to the rats in the control group (SHAM), whereas no significant changes in trabecular thickness were found (Tb.Th).

Kalsis treatment for 3 months (OVX + K25) partially avoided a decrease in BV/TV and in Tb.N, as evidenced by the ovariectomized rats (OVX). Also, the preventive Kalsis treatment administered immediately after ovariectomy $(\mathrm{OVX}+\mathrm{K} 25)$ avoided an increase in Tb.Sp, albeit not completely. Kalsis produced a significant increase in Tb.Th with respect to the SHAM and OVX groups.

The results of the trabecular pattern factor (Tb.Pf) show that ovariectomy produced a disconnection of the trabecular structure when compared with the SHAM group. Kalsis treatment attenuated this effect and partially maintained the trabecular connection.
TABLE 3: 3D trabecular microarchitecture analysis of femur by microtomography in rats which were sham operated (SHAM), ovariectomized (OVX), or ovariectomized and treated with kalsis $(\mathrm{OVX}+\mathrm{K} 25)$. The parameters studied are bone volume/total volume (BV/TV; \%), trabecular number (Tb.N; 1/mm), trabecular thickness (Tb.Th; mm), trabecular separation (Tb.Sp; mm), and trabecular pattern factor (Tb.Pf; $1 / \mathrm{mm}$ ). Female Wistar rats, 6 months old at the beginning of the study, ovariectomized (OVX) or sham-operated (SHAM) and randomized in the following groups: SHAM group (SHAM) treated with vehicle for 3 months; ovariectomized group (OVX) also treated with vehicle for 3 month; ovariectomized group treated with Kalsis at a dose of $25 \mathrm{mg} / \mathrm{kg} /$ day, for 3 months (OVX + K25). Statistical significance $P<0.05$ : a vresus SHAM; b versus OVX.

\begin{tabular}{lccc}
\hline & SHAM & OVX & OVX + K25 \\
\hline BV/TV $(\%)$ & $32.11 \pm 6.68$ & $13.47 \pm 4.88^{\mathrm{a}}$ & $20.21 \pm 4.15^{\mathrm{a}, \mathrm{b}}$ \\
Tb.N $(1 / \mathrm{mm})$ & $4.55 \pm 0.80$ & $1.82 \pm 0.53^{\mathrm{a}}$ & $2.24 \pm 0.15^{\mathrm{a}, \mathrm{b}}$ \\
Tb.Th $(\mathrm{mm})$ & $0.073 \pm 0.004$ & $0.072 \pm 0.007$ & $0.090 \pm 0.020^{\mathrm{a}, \mathrm{b}}$ \\
Tb.Sp $(\mathrm{mm})$ & $0.152 \pm 0.036$ & $0.499 \pm 0.127^{\mathrm{a}}$ & $0.405 \pm 0.053^{\mathrm{a}, \mathrm{b}}$ \\
Tb.Pf $(1 / \mathrm{mm})$ & $-5.571 \pm 5.521$ & $7.575 \pm 5.411^{\mathrm{a}}$ & $2.414 \pm 3.882^{\mathrm{a}, \mathrm{b}}$ \\
\hline
\end{tabular}

\section{Discussion}

In this study, we found that daily treatment of rats for 3 months using a dietary supplement (Kalsis) that contains vitamin $C$, vitamin $E$, and selenium prevented loss of FBMD and LBMD due to ovariectomy. Ovariectomized rats treated with Kalsis presented FBMD and LBMD levels similar to those of SHAM group. Untreated OVX rats showed a significant decrease both in LBMD and FBMD with respect to control animals. The loss of bone mass due to ovariectomy has been widely reported in previous works $[25,33]$.

When studying variations in bone mass through trabecular microarchitecture analysis of the femur using microtomography, we did not find a complete preservation of microarchitecture with Kalsis treatment. The decrease in BV/TV and Tb.N and the increase in Tb.Sp with respect to the SHAM group were lower in the OVX group treated with Kalsis than in the untreated group. Moreover, treatment with Kalsis brought about a significant increase in $\mathrm{Tb}$.Th with respect to the SHAM and OVX groups. Tb.Pf also was maintained in the OVX $+\mathrm{K} 25$ group values between the SHAM and untreated castrated rats. These results show that, used as a preventive treatment, Kalsis attenuates bone loss produced by ovariectomy to such an extent that differences in bone mass between OVX-treated and intact rats can only be detected by $\mu \mathrm{CT}$, and not by conventional measurement of BMD by DEXA. According to the detection of differences in $\mu \mathrm{CT}$ but not in $\mathrm{BMD}$, previous studies demonstrated that trabecular area and BV/TV determinations were more sensitive than BMD [34].

When we analyzed the action of Kalsis on bone remodelling by examining biochemical markers of bone turnover, we observed the same observations as other authors had $[33,35]$, namely an increase in bone formation (BGP and PINP) and in bone resorption (CTX/TRAP) in OVX rats. As PINP has only been recently available for its use in rats, few reports exist in the literature about its use in the 
SHAM
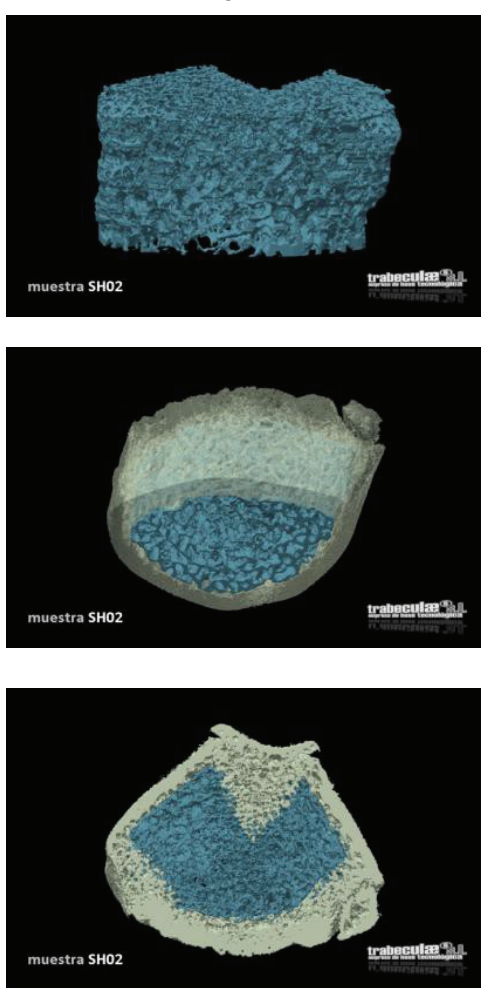

OVX
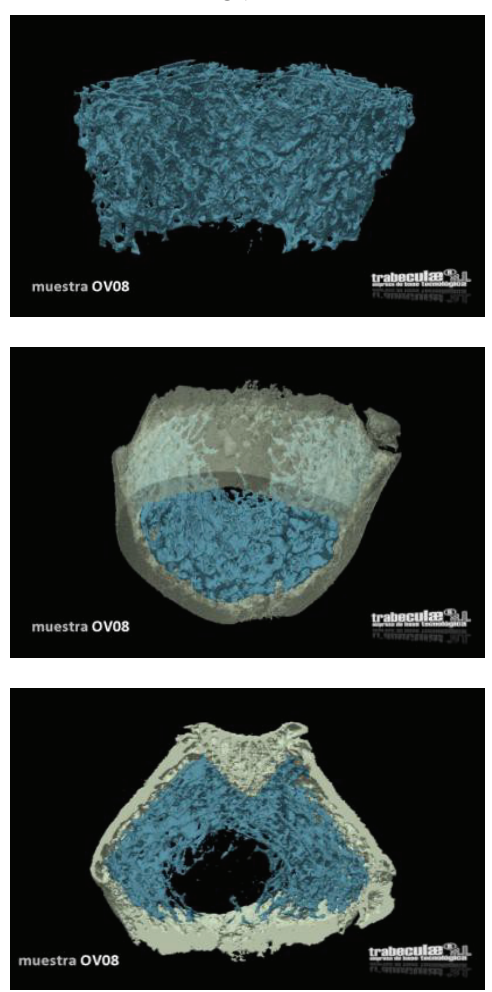

OVX + K25
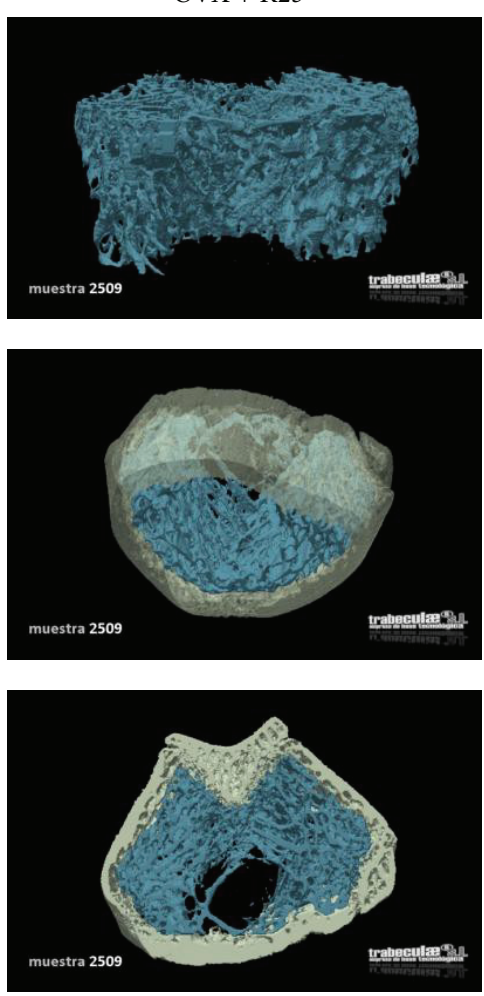

FIGURE 1: Representative images of bone trabecular 3D-microarchitecture in femur sections of all the study groups obtained by computed microtomography. The study groups of female Wistar rats, 6 months old at the beginning of the study, are ovariectomized (OVX) or shamoperated (SHAM) and randomized in the following groups: SHAM group (SHAM) treated with vehicle for 3 months; ovariectomized group (OVX) also treated with vehicle for 3 months; ovariectomized group treated with Kalsis at a dose of $25 \mathrm{mg} / \mathrm{kg} / \mathrm{day}$, for $3 \mathrm{months}$ (OVX + K25).

study of bone remodelling in these animals. One of them is that of Rissanen et al. [36] who observed a significant increase in PINP levels after ovariectomy. A recent study demonstrated that secreted TRAP $5 b$ is a reliable marker of osteoclast number and secreted CTX is a reliable marker of the resorbing activity of osteoclasts [37]. Because resorption and therefore the total activity of osteoclasts are increased after OVX, and owing to the fact that the absolute number of osteoclasts is decreased, the remaining osteoclasts must be substantially more active. Osteoclast activity can be conveniently calculated by dividing the results obtained with a reliable marker of osteoclast activity, such as CTX, by the results obtained with a reliable marker of osteoclast number, such as TRAP 5b. Therefore, the ratio of CTX/TRAP 5b appears to be an extremely useful parameter in the rat OVX model, where osteoclast activity is increased while osteoclast number is decreased [37]. This is the reason why we used CTX/TRAP $5 b$ as an index of bone resorption rather than individual values of both biochemical markers. The levels of biochemical markers of bone formation and resorption were similar to those of the ovariectomized animals not receiving treatment. So, it can be concluded that Kalsis treatment did not produce any effect on the increase of bone remodelling due to ovariectomy. These results suggest that Kalsis works not by causing variations in bone remodelling but rather through the antioxidant action of some of its components, possibly mainly through the action of selenium. The synergistic action of ascorbic acid and vitamin E cannot be excluded.

In this respect, there are recent works that show that association between selenium and vitamin $\mathrm{E}$ reduces prostate cancer incidence through changes in the oxidation-reduction balance of cells, decreasing expression of antiapoptotic and proinflammatory genes, that are associated with prostate cancer and enhancing DNA repair [38]. This changes in the oxidation-reduction balance of cells could also affect bone health as has been demonstrated in previously referenced works, and this could be the mechanism of action of Kalsis (that contains the antioxidants ascorbic acid, vitamin $\mathrm{E}$ and selenium) on osseous tissue.

According to an internal publication edited by Catalysis laboratories [26], the components of Kalsis were subjected to a molecular activation process that increases significantly the antioxidant action of this compound. Although, individually, the different antioxidants components of Kalsis could produce a positive effect on the red-ox status of bone cells, the activation process seems to be essential to obtain the greatest biological activity in the treatment of diseases which directly or indirectly produce free radicals [26]. In this respect, the manufacturer shows in his publication [26] the example 
of Viusid, an antioxidant supplement with ascorbic acid, zinc, and glycyrrhizic acid. After the activation process, this compound showed an antioxidant capacity (analyzed by Somogyi-Nelson reagent) 7.8 times higher than in its original nonactive state. There are recent works with other antioxidant preparations from Catalysis laboratories, treated with the same activation procedure as Kalsis, that demonstrate their different positive effects on bone health [32, 39-41]. However, there are no previous works about Kalsis action, although several studies in humans seem to demonstrate its beneficial effects in other different pathologies (unpublished results).

Another interesting advantage of administering this compound may be weight gain. It is known that there is a net body gain weight after menopause, as can be observed in the group of OVX rats. Although the rats treated with Kalsis had a greater weight gain than the control group, this gain was significantly lower than that of the untreated OVX group.

In conclusion, administration of Kalsis, a food supplement containing ascorbic acid, vitamin E, and selenium which is submitted to a molecular activation process by the manufacturer, partially prevented bone loss in ovariectomized rats; bone loss was undetectable by classical densitometric methods like DEXA and could only be observed by using more sensitive methods like computed microtomography. This compound also partially prevented the weight gain produced by ovariectomy. The mechanism of action of this supplement is not the result of a decrease in the rate of bone remodelling, because levels of bone formation and resorption are similar to those of untreated animals. The antioxidant action of this compound as a cause of its beneficial effect is suggested. Further work will be necessary, measuring markers of oxidation stress in ovariectomized rats with and without Kalsis treatment.

Due to the fact that some risk factors for osteoporosis, such as smoking and diabetes mellitus, are associated with increased oxidative stress, human studies are needed to analyze the possible role played by Kalsis in preventing the development of osteoporosis in humans with multiple risk factors. It may be especially beneficial to implement this treatment in the periods in which drugs commonly used to treat osteoporosis need to be suspended due to the risk of negative effects due to their prolonged use.

\section{Acknowledgments}

This work was supported by a grant from Catalysis Laboratories (Spain).

\section{References}

[1] "NIH Consensus development panel on osteoporosis prevention $\mathrm{D}$ and $\mathrm{T}$. Osteoporosis prevention, diagnosis, and therapy," Journal of the American Medical Association, vol. 285, pp. 785-795, 2001.

[2] R. Eastell, Primer on the Metabolic Bone Diseases and Disorders of Mineral Metabolism, John Wiley \& Sons, Hoboken, NJ, USA, 5th edition, 2003.
[3] S. Srivastava, M. N. Weitzmann, S. Cenci, F. P. Ross, S. Adler, and R. Pacifici, "Estrogen decreases TNF gene expression by blocking JNK activity and the resulting production of c-Jun and JunD," Journal of Clinical Investigation, vol. 104, no. 4, pp. 503-513, 1999.

[4] S. Srivastava, G. Toraldo, M. N. Weitzmann, S. Cenci, F. P. Ross, and R. Pacifici, "Estrogen decreases osteoclast formation by down-regulating receptor activator of NF-kappa B ligand (RANKL)-induced JNK activation," Journal of Biological Chemistry, vol. 276, no. 12, pp. 8836-8840, 2001.

[5] S. Kousteni, L. Han, J. R. Chen et al., "Kinase-mediated regulation of common transcription factors accounts for the boneprotective effects of sex steroids," Journal of Clinical Investigation, vol. 111, no. 11, pp. 1651-1664, 2003.

[6] M. N. Weitzmann and R. Pacifici, "Estrogen deficiency and bone loss: an inflammatory tale," Journal of Clinical Investigation, vol. 116, no. 5, pp. 1186-1194, 2006.

[7] S. Basu, K. Michaëlsson, H. Olofsson, S. Johansson, and H. Melhus, "Association between oxidative stress and bone mineral density," Biochemical and Biophysical Research Communications, vol. 288, no. 1, pp. 275-279, 2001.

[8] D. Maggio, M. Barabani, M. Pierandrei et al., "Marked decrease in plasma antioxidants in aged osteoporotic women: results of a cross-sectional study," Journal of Clinical Endocrinology and Metabolism, vol. 88, no. 4, pp. 1523-1527, 2003.

[9] S. Muthusami, I. Ramachandran, B. Muthusamy et al., "Ovariectomy induces oxidative stress and impairs bone antioxidant system in adult rats," Clinica Chimica Acta, vol. 360, no. 1-2, pp. 81-86, 2005.

[10] M. C. Polidori, W. Stahl, O. Eichler, I. Niestroj, and H. Sies, "Profiles of antioxidants in human plasma," Free Radical Biology and Medicine, vol. 30, no. 5, pp. 456-462, 2001.

[11] K. Rahman, "Studies on free radicals, antioxidants, and cofactors," Clinical Interventions in Aging, vol. 2, no. 2, pp. 219236, 2007.

[12] J. M. Lean, C. J. Jagger, B. Kirstein, K. Fuller, and T. J. Chambers, "Hydrogen peroxide is essential for estrogendeficiency bone loss and osteoclast formation," Endocrinology, vol. 146, no. 2, pp. 728-735, 2005.

[13] G. N. Schrauzer, "Anticarcinogenic effects of selenium," Cellular and Molecular Life Sciences, vol. 57, no. 13-14, pp. 1864-1873, 2000.

[14] G. P. Trueba, G. M. Sánchez, and A. Giuliani, "Oxygen free radical and antioxidant defense mechanism in cancer," Frontiers in Bioscience, vol. 9, pp. 2029-2044, 2004.

[15] R. Moreno-Reyes, D. Egrise, J. Nève, J. L. Pasteels, and A. Schoutens, "Selenium deficiency-induced growth retardation is associated with an impaired bone metabolism and osteopenia," Journal of Bone and Mineral Research, vol. 16, no. 8, pp. 1556-1563, 2001.

[16] M. Kleerekoper, "Osteoporosis prevention and therapy: preserving and building strength through bone quality," Osteoporosis International, vol. 17, no. 12, pp. 1707-1715, 2006.

[17] D. Goltzman, "Discoveries, drugs and skeletal disorders," Nature Reviews Drug Discovery, vol. 1, no. 10, pp. 784-796, 2002.

[18] P. Vestergaard, N. R. Jorgensen, L. Mosekilde, and P. Schwarz, "Effects of parathyroid hormone alone or in combination with antiresorptive therapy on bone mineral density and fracture risk-a meta-analysis," Osteoporosis International, vol. 18, no. 1, pp. 45-57, 2007. 
[19] P. J. Meunier, D. O. Slosman, P. D. Delmas et al., "Strontium ranelate: dose-dependent effects in established postmenopausal vertebral osteoporosis-a 2-year randomized placebo controlled trial," Journal of Clinical Endocrinology and Metabolism, vol. 87, no. 5, pp. 2060-2066, 2002.

[20] S. C. Chai, C. I. Wei, K. Brummel-Smith, and B. H. Arjmandi, "The role of vitamin E in reversing bone loss," Aging Clinical and Experimental Research, vol. 20, no. 6, pp. 521-527, 2008.

[21] Z. Yang, Z. Zhang, K. L. Penniston, N. Binkley, and S. A. Tanumihardjo, "Serum carotenoid concentrations in postmenopausal women from the United States with and without osteoporosis," International Journal for Vitamin and Nutrition Research, vol. 78, no. 3, pp. 105-111, 2008.

[22] C. L. Shen, P. Wang, J. Guerrieri, J. K. Yeh, and J. S. Wang, "Protective effect of green tea polyphenols on bone loss in middle-aged female rats," Osteoporosis International, vol. 19, no. 7, pp. 979-990, 2008.

[23] C.-L. Shen, J. K. Yeh, J. J. Cao, and J.-S. Wang, "Green tea and bone metabolism," Nutrition Research, vol. 29, no. 7, pp. 437 456, 2009.

[24] D. J. Morton, E. L. Barrett-Connor, and D. L. Schneider, "Vitamin C supplement use and bone mineral density in postmenopausal women," Journal of Bone and Mineral Research, vol. 16, no. 1, pp. 135-140, 2001.

[25] M. D. Curiel, J. A. Calero, R. Guerrero, J. Gala, R. Gazapo, and C. De la Piedra, "Effects of LY-117018 $\mathrm{HCl}$ on bone remodeling and mineral density in the oophorectomized rat," American Journal of Obstetrics and Gynecology, vol. 178, no. 2, pp. 320-325, 1998.

[26] A. González, Antioxidants: Study and Applications, Internal Editions of Catalysis Laboratories, 2005.

[27] J. G. Paniagua, M. Díaz-Curiel, C. De La Piedra Gordo, C. C. Reparaz, and M. T. García, "Bone mass assessment in rats by dual energy X-ray absorptiometry," British Journal of Radiology, vol. 71, pp. 754-758, 1998.

[28] I. A. Feldkamp, L. C. Davis, and J. W. Kress, "Practical conebeam algorithm," Journal of the Optical Society of America A, vol. 1, no. 6, pp. 612-619, 1984.

[29] T. Hildebrand and P. Rüegsegger, "A new method for the model-independent assessment of thickness in threedimensional images," Journal of Microscopy, vol. 185, no. 1, pp. 67-75, 1997.

[30] D. Ulrich, B. Van Rietbergen, A. Laib et al., "The ability of three-dimensional structural indices to reflect mechanical aspects of trabecular bone," Bone, vol. 25, no. 1, pp. 55-60, 1999.

[31] M. Hahn, M. Vogel, M. Pompesius-Kempa, and G. Delling, "Trabecular bone pattern factor-a new parameter for simple quantification of bone microarchitecture," Bone, vol. 13, no. 4, pp. 327-330, 1992.

[32] J. Hernández and D. Vargas, "Utilidad de Diamel en pacientes con dibetes mellitus tipo 2 en tratmiento combinado con glibenclamida," Avances en Diabetología, vol. 23, pp. 49-55, 2007.

[33] A. F. Stewart, R. L. Cain, D. B. Burr, D. Jacob, C. H. Turner, and J. M. Hock, "Six-month daily administration of parathyroid hormone and parathyroid hormone-related protein peptides to adult ovariectomized rats markedly enhances bone mass and biomechanical properties: a comparison of human parathyroid hormone 1-34, parathyroid hormone-related protein 1-36, and SDZ- parathyroid hormone 893," Journal of Bone and Mineral Research, vol. 15, no. 8, pp. 1517-1525, 2000.
[34] R. G. Erben, J. Eberle, K. Stahr, and M. Goldberg, "Androgen deficiency induces high turnover osteopenia in aged male rats: a sequential histomorphometric study," Journal of Bone and Mineral Research, vol. 15, no. 6, pp. 1085-1098, 2000.

[35] M. Kneissel, A. Boyde, and J. A. Gasser, "Bone tissue and its mineralization in aged estrogen-depleted rats after long-term intermittent treatment with parathyroid hormone (PTH) analog SDZ PTS 893 or human PTH(1-34)," Bone, vol. 28, no. 3, pp. 237-250, 2001.

[36] J. P. Rissanen, M. I. Suominen, Z. Peng et al., "Shortterm changes in serum PINP predict long-term changes in trabecular bone in the rat ovariectomy model," Calcified Tissue International, vol. 82, no. 2, pp. 155-161, 2008.

[37] J. P. Rissanen, M. I. Suominen, Z. Peng, and J. M. Halleen, "Secreted tartrate-resistant acid phosphatase $5 \mathrm{~b}$ is a marker of osteoclast number in human osteoclast cultures and the rat ovariectomy model," Calcified Tissue International, vol. 82, no. 2, pp. 108-115, 2008.

[38] D. Tsavachidou, T. J. McDonnell, S. Wen et al., "Selenium and vitamin E: cell type- and intervention-specific tissue effects in prostate cancer," Journal of the National Cancer Institute, vol. 101, no. 5, pp. 306-320, 2009.

[39] E. Vilar Gomez, A. Rodriguez De Miranda, B. Gra Oramas et al., "Clinical trial: a nutritional supplement Viusid, in combination with diet and exercise, in patients with nonalcoholic fatty liver disease," Alimentary Pharmacology and Therapeutics, vol. 30, no. 10, pp. 999-1009, 2009.

[40] D. Uddin and M. A. M. I. Islam, "Findings of the 3month supportive treatment with oncoxin solution beside the standard modalities of patients with different neoplastic diseases," TAJ, vol. 22, pp. 172-175, 2009.

[41] E. V. Gomez, Y. M. Perez, H. V. Sanchez et al., "Antioxidant and immunomodulatory effects of Viusid in patients with chronic hepatitis C," World Journal of Gastroenterology, vol. 16, no. 21, pp. 2638-2647, 2010. 


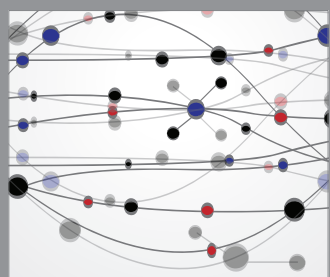

The Scientific World Journal
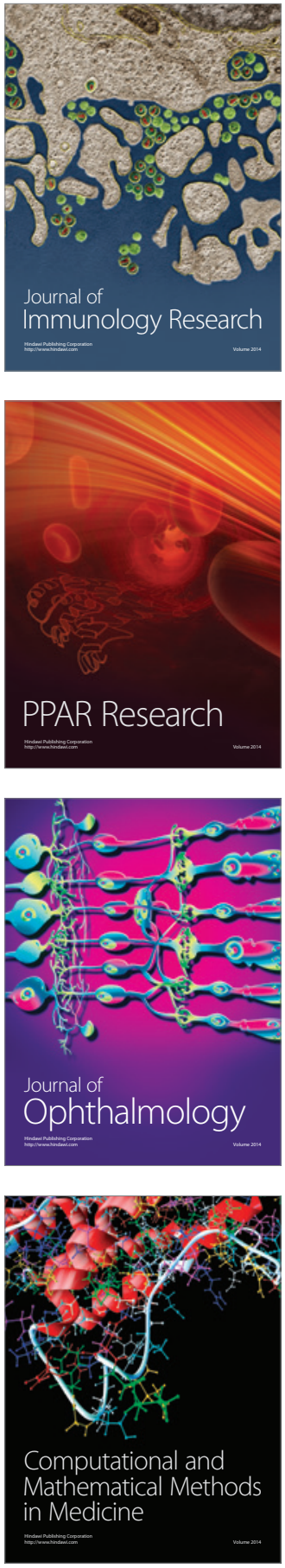

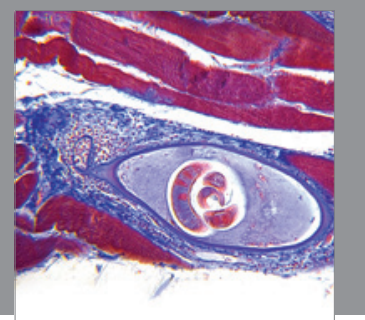

Gastroenterology

Research and Practice
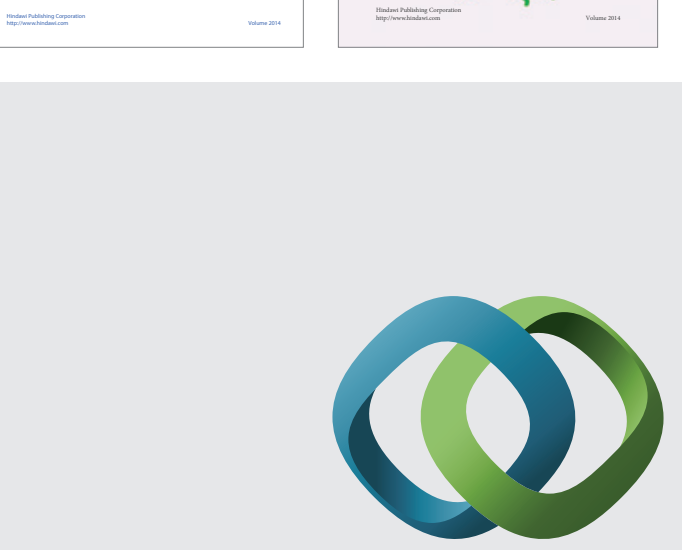

\section{Hindawi}

Submit your manuscripts at

http://www.hindawi.com
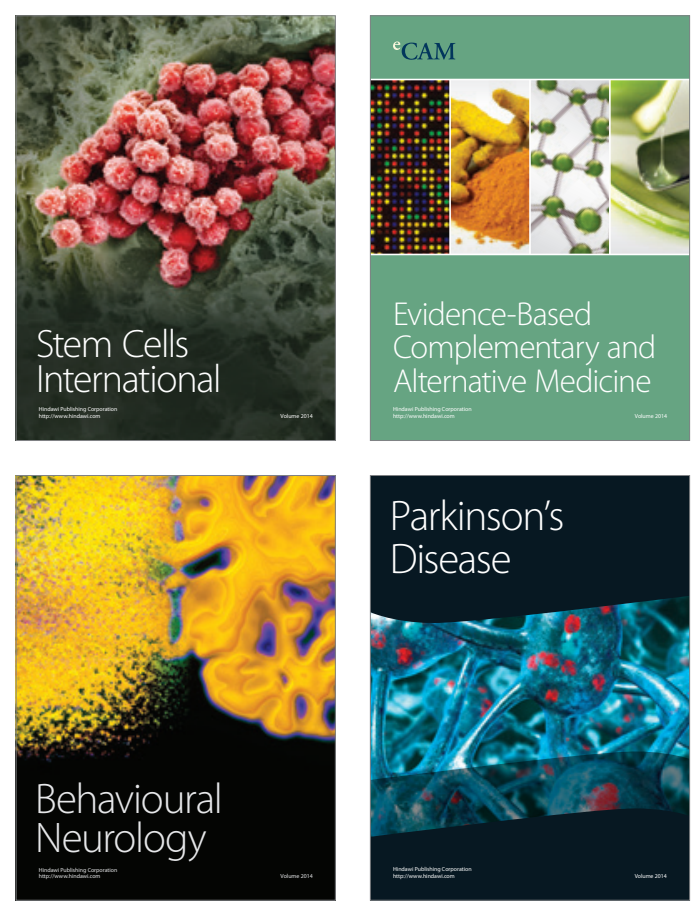

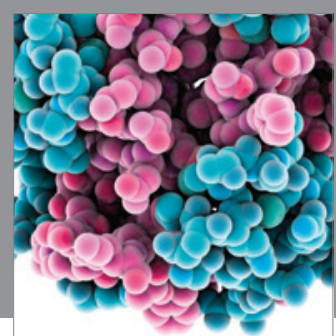

Journal of
Diabetes Research

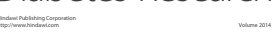

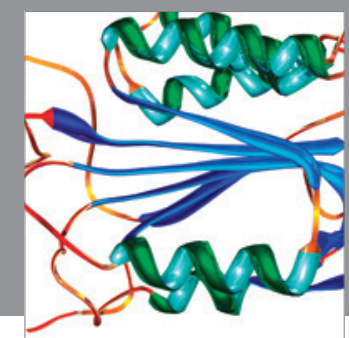

Disease Markers
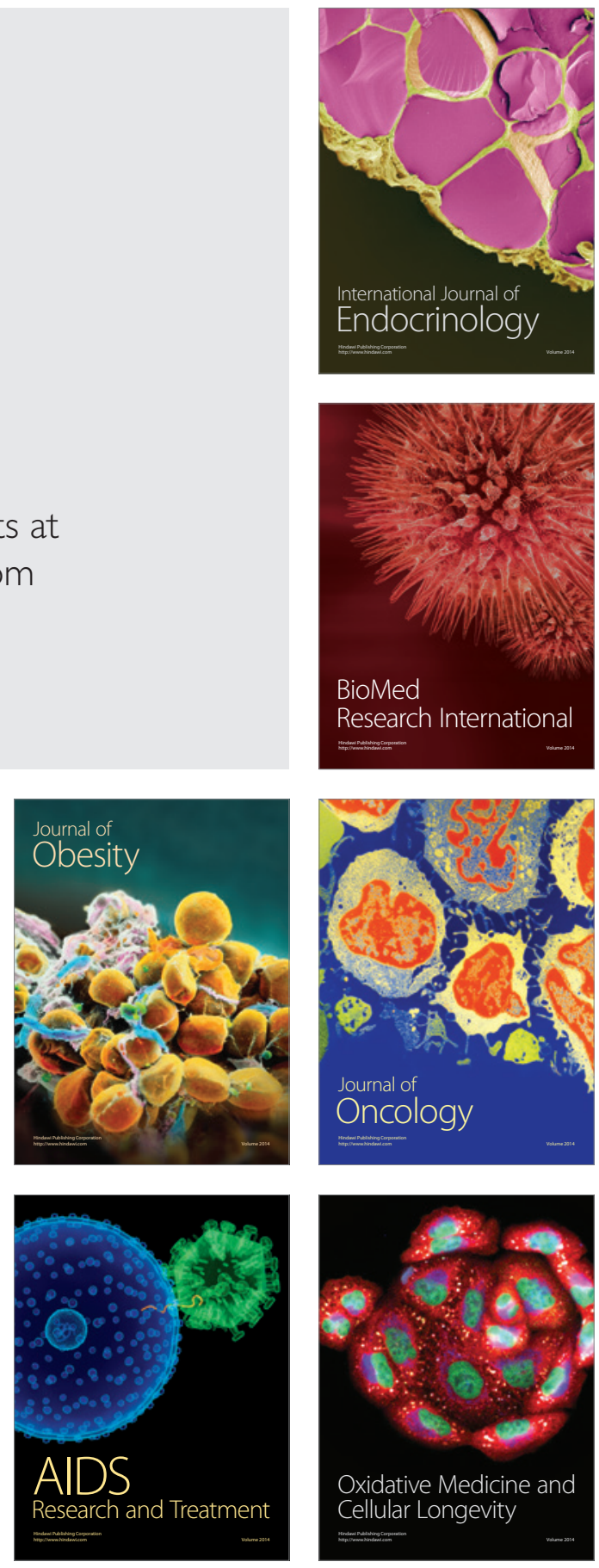